\title{
MeQTL analysis of childhood obesity links epigenetics with a risk SNP rs17782313 near MC4R from meta-analysis
}

\author{
Yuping Tang ${ }^{1, *}$, Bo Jinn ${ }^{2, *}$, Lingling Zhou ${ }^{1, *}$, Weifeng $\mathbf{L u}^{3}$ \\ ${ }^{1}$ Department of Orthopaedic, Children's Hospital of Nanjing Medical University, Nanjing, China \\ ${ }^{2}$ Department of Neurology, Children's Hospital of Nanjing Medical University, Nanjing, China \\ ${ }^{3}$ Surgical Intensive Care Unite, Children's Hospital of Nanjing Medical University, Nanjing, China \\ *These authors contributed equally to this work
}

Correspondence to: Weifeng Lu, email: NJWeifeng_lu@163.com

Keywords: rs17782313, MC4R, meQTL, eQTL, childhood obesitys

Received: September 22, $2016 \quad$ Accepted: November 23, 2016

Published: December 01, 2016

\section{ABSTRACT}

Earlier GWAS has identified that rs17782313 near MC4R was associated with obesity. However, subsequent studies showed conflicting results, especially among childhood. Besides, the mechanisms underlying the association between rs17782313 and childhood obesity remain largely unexplored, and genetic and epigenetic may interact and together affect the development of childhood obesity. We conducted a comprehensive meta-analysis to assess the association between rs17782313 and childhood obesity. MeQTL and eQTL analysis was applied to explore the effect of rs17782313 on DNA methylation and MC4R expression. We found that rs17782313 near MC4R was associated with increased childhood obesity risk and BMI z-score in several inheritable models $(P<0.05)$. Additionally, the similar trend was observed among subgroups of Asians, Caucasian. Furthermore, meQTL and eQTL analysis indicated that individuals carrying rs17782313 TT genotype were significantly associated with increased methylation level of cg10097150 located in MC4R promoter and decreased expression of MC4R than those with CT/CC genotype $\left(P=1.7 \times 10^{-4}\right.$ and $P=1.9 \times 10^{-3}$ respectively). Our results strongly confirmed that rs 17782313 was associated with increased risk of childhood obesity. Furthermore, rs17782313 T allele was correlated with promoter hypermethylation and decreased expression of MC4R, thus involved in the development of childhood obesity.

\section{INTRODUCTION}

Globally, obesity is becoming an increasingly serious clinical and public health challenge and is a major risk factor for various chronic diseases, such as cardiovascular diseases, type 2 diabetes, metabolic syndrome and several types of cancers $[1,2]$. The prevalence of obesity is up to $25 \%$ in western country and increasing in children $[3,4]$. Emerging evidence suggests that childhood obesity resulted in an increased mortality in adults [5]. Environmental factors, especially lifestyle and excessive food intake, are closely related to epidemic obesity. However, multiple twin studies have suggested that genetic factors explain about $65 \%$ of the variance in obesity $[6,7]$. Genome-wide association studies (GWASs) have identified a number of loci associated with body mass index (BMI), which is a conventional measure of obesity $[8,9]$. However, the biological functions of most well known genetic association with small effect size have not fully explained. The majority of GWAS loci are located within non-coding or intergenic regions and do not alter gene coding sequence $[10,11]$. It is assumed that GWAS polymorphisms are located in regulatory elements and influence the disease by changing gene expression, which is called expression quantitative trait loci (eQTL) $[12,13]$.

Recently, it is becoming distinct that epigenetics, especial DNA methylation, also play a key role in obesity pathogenesis. Mounting evidences showed that the SNPs can affect methylation level at nearby $\mathrm{CpG}$ sites [14]. Such SNPs are known as methylation quantitative trait loci (meQTLs). The identification of meQTLs may assist in identifying novel candidate disease-associated genes and 
providing a new approach for connecting DNA sequence variation with phenotype [10].

Previous GWAS revealed that rs17782313 near the melanocortin 4 receptor $(M C 4 R)$ gene was associated with increased risk of obesity in Europeans [15]. It is well established that MC4R plays a key role in coordinating energy homeostasis and body weight [16]. However, subsequent studies from different age groups have showed conflicting and inconclusive results, especially among childhood. Meanwhile, little is known of the molecular biological mechanisms underlying the effect of rs17782313 on the development of childhood obesity.

In this study, we applied a comprehensive metaanalysis to make an accurate assessment of the association of the MC4R rs17782313 with childhood obesity risk. Furthermore, most previous studies have investigated genetic and epigenetic mechanisms independently and their interaction was not considered. Our study aimed to combine genetic and epigenetic together to investigate the mechanisms that rs17782313 involved in the development of childhood obesity by using public Gene Expression Omnibus (GEO) and The Cancer Genome Atlas (TCGA) databases.

\section{RESULTS}

\section{Meta-analysis result}

A total of twelve articles including 5,908 cases of obesity and 19,826 controls were enrolled in our metaanalysis study. The detailed characteristics of studies included in this meta-analysis study are supplied in Table 1 . The result showed that rs 17782313 was significantly associated with childhood obesity in all inheritable models (homozygous model: $\mathrm{OR}=1.84,95 \% \mathrm{CI}=1.44-2.36$; heterozygous model: $\mathrm{OR}=1.48,95 \% \mathrm{CI}=1.29-1.70$; dominant model: $\mathrm{OR}=1.35,95 \% \mathrm{CI}=1.19-1.55$; recessive model: $\mathrm{OR}=1.67,95 \% \mathrm{CI}=1.39-1.99)$ (Table 2; Figure 1A). In the stratification analysis, an obvious association was observed between rs17782313 polymorphism and childhood obesity in subgroup of Caucasian and Asian in all inheritable models $(P<0.05)$. Moreover, the similar significant results were also found among subgroups of Taqman and other genotyping methods and sample size more than 1000, but not in the subgroup of sample size less than 1000 under the heterozygous model and recessive model (Table 2). Besides, we applied metaanalysis to evaluate the relationship between rs17782313 genotype and BMI z-score, and four articles were enrolled in the statistical analysis. The results indicated that rs $17782313 \mathrm{~T}>\mathrm{C}$ polymorphism was notably associated with childhood BMI z-score (WMD $=0.14,95 \% \mathrm{CI}$ $=0.06-0.23$ ) (Figure 1B). In addition, we obtained a boundary significant result in the replication of public GEO (GSE73103) database with limited samples (dominant model: $\mathrm{OR}=1.24,95 \% \mathrm{CI}=1.01-1.62, P=0.049)$.

\section{The impact of rs17782313 on $M C 4 R$ expression}

To investigate eQTL, we analyzed data from The Cancer Genome Atlas (TCGA) for whom both germline genotype from Affymetrix Genome-Wide Human SNP Array 6.0 and mRNA expression in intestines normal tissues from Illumina HiSeq 2000 RNA Sequencing Version 2 were available. ANOVA result revealed that individuals carrying rs17782313 TT genotypes were associated with decreased the expression of $M C 4 R$ than those with $\mathrm{CT} / \mathrm{CC}$ genotypes $\left(P=1.9 \times 10^{-3}\right)$. (Figure 2).

\section{Association between rs17782313 polymorphism with DNA methylation}

Genotype data and correspondent methylation level data were available, which were requested from public GEO database (GSE73103). We extract CpG methylation probes located in $500 \mathrm{~kb}$ upstream and downstream from rs17782313. Associations between rs17782313 and methylation levels were tested using a linear model adjusted for age, sex, weight category and a proxy for blood cell type counts. Interestingly, we found that rs 17782313 was significantly correlated with methylation level of $\operatorname{cg} 10097150$ which is located in the promoter of $M C 4 R\left(P=1.7 \times 10^{-4}\right)$. Here, Individuals with the rs17782313 TT genotype have an obvious hypermethylation level in $M C 4 R$ promoter than those with $\mathrm{CT} / \mathrm{CC}$ genotype (Figure 2).

\section{DISCUSSION}

Recently, the GWAS have identified rs17782313 was associated with BMI and obesity in Caucasians. However, the relationship of rs17782313 with obesity among other ethnicities are not fully explored, especially in children. In this study, the results of meta-analysis strongly confirmed that rs17782313 near MC4R gene was significantly associated with increased risk of childhood obesity in four inheritable models (homozygous model: $\mathrm{OR}=1.84,95 \% \mathrm{CI}=1.44-2.36$; heterozygous model: $\mathrm{OR}$ $=1.48,95 \% \mathrm{CI}=1.29-1.70$; dominant model: $\mathrm{OR}=1.35$, $95 \% \mathrm{CI}=1.19-1.55$; recessive model: $\mathrm{OR}=1.67,95 \% \mathrm{CI}$ $=1.39-1.99)$. Further stratification analyses showed that the similar trend association was also observed in subgroup of Asians, Caucasian, sample size more than 1000. Besides, MC4R rs 17782313 was boundary correlated with childhood obesity in the replication of available public GEO database (dominant model: $\mathrm{OR}=1.24,95 \% \mathrm{CI}=$ $1.01-1.62, P=0.049)$. In addition, another meta-analysis indicated that $\mathrm{rs} 17782313 \mathrm{~T}>\mathrm{C}$ polymorphism was notably associated with childhood BMI z-score (WMD = $0.14,95 \% \mathrm{CI}=0.06-0.23$ ).

SNP rs17782313 mapped $188 \mathrm{~kb}$ downstream of $M C 4 R$, which is well established as a candidate gene for obesity. $M C 4 R$ encoded a membrane-bound receptor 
Table 1: Main characteristics of studies included in this Meta-analysis study

\begin{tabular}{llllllll}
\hline Study & Publication year & Country & Ethnicity & Genotyping method & Sample size & Cases & Controls \\
\hline Judith & 2014 & Chilean & Mixed & TaqMan & $<1000$ & 238 & 139 \\
Vasan & 2013 & India & Asian & TaqMan & $\geq 1000$ & 175 & 998 \\
Dwivedi & 2013 & India & Asian & iPLEX & $\geq 1000$ & 425 & 904 \\
Dwivedi & 2013 & India & Asian & iPLEX & $\geq 1000$ & 157 & 904 \\
Zhao & 2013 & China & Asian & TaqMan & $<1000$ & 371 & 394 \\
Xi & 2011 & China & Asian & TaqMan & $\geq 1000$ & 1,229 & 1,619 \\
Vogel & 2011 & America & Caucasian & TaqMan & $\geq 1000$ & 881 & 434 \\
Wu & 2010 & China & Asian & TaqMan & $\geq 1000$ & 1,207 & 1,589 \\
Wu & 2010 & China & Asian & TaqMan & $\geq 1000$ & 648 & 1,589 \\
Cauchi & 2009 & Finnish & Caucasian & TaqMan & $\geq 1000$ & 148 & 3,802 \\
Cauchi & 2009 & Finnish & Caucasian & TaqMan & $\geq 1000$ & 177 & 3,392 \\
Cauchi & 2009 & Finnish & Caucasian & TaqMan & $\geq 1000$ & 252 & 4,062 \\
\hline
\end{tabular}

Table 2: Meta-analysis of association between $M C 4 R$ polymorphism and childhood obesity risk

\begin{tabular}{|c|c|c|c|c|c|c|c|c|c|}
\hline \multirow{2}{*}{ Variables } & \multirow{2}{*}{$n$} & \multicolumn{2}{|l|}{ CC vs. TT } & \multicolumn{2}{|l|}{ CT vs. TT } & \multicolumn{2}{|l|}{ CC vs. TT/CT } & \multicolumn{2}{|l|}{ CT/CC vs. TT } \\
\hline & & OR $(95 \% \mathrm{CI})$ & $\boldsymbol{P}_{\text {het }}$ & OR $(95 \% \mathrm{CI})$ & $P_{h e t}$ & OR $(95 \% \mathrm{CI})$ & $P_{h e t}$ & OR $(95 \%$ CI $)$ & $\boldsymbol{P}_{\text {het }}$ \\
\hline Total & 12 & $1.84(1.44-2.36)$ & 0.001 & $1.48(1.29-1.70)$ & 0.756 & $1.67(1.39-1.99)$ & 0.082 & $1.35(1.19-1.55)$ & 0.000 \\
\hline \multicolumn{10}{|l|}{ Ethnicity } \\
\hline Asian & 7 & $1.95(1.39-2.74)$ & 0.000 & $1.52(1.30-1.77)$ & 0.561 & $1.71(1.34-2.18)$ & 0.015 & $1.38(1.13-1.68)$ & 0.000 \\
\hline Caucasian & 4 & $1.80(1.31-2.46)$ & 0.611 & $1.38(1.00-1.90)$ & 0.687 & $1.61(1.17-2.20)$ & 0.682 & $1.33(1.14-1.55)$ & 0.319 \\
\hline Mixed & 1 & $1.06(0.25-4.52)$ & - & $0.72(0.16-3.25)$ & - & $0.97(0.23-4.13)$ & - & $1.43(0.87-2.35)$ & - \\
\hline \multicolumn{10}{|c|}{ Genotyping method } \\
\hline TaqMan & 10 & $1.66(1.26-2.19)$ & 0.007 & $1.49(1.27-1.75)$ & 0.586 & $1.58(1.26-1.99)$ & 0.055 & $1.26(1.13-1.41)$ & 0.025 \\
\hline Others & 2 & $2.72(2.06-3.59)$ & 0.784 & $1.46(1.13-1.89)$ & 0.926 & $1.92(1.51-2.45)$ & 0.859 & $2.07(1.68-2.54)$ & 0.776 \\
\hline \multicolumn{10}{|l|}{ Sample size } \\
\hline$\geq 1000$ & 10 & $1.81(1.39-2.36)$ & 0.001 & $1.47(1.27-1.69)$ & 0.812 & $1.64(1.36-1.97)$ & 0.068 & $1.35(1.16-1.57)$ & 0.000 \\
\hline$<1000$ & 2 & $2.36(1.26-4.43)$ & 0.232 & $1.86(0.98-3.53)$ & 0.178 & $1.97(0.83-4.69)$ & 0.230 & $1.39(1.08-1.78)$ & 0.904 \\
\hline
\end{tabular}

and member of the melanocortin receptor family protein. Multiple lines of studies suggested that MC4R might be involved in regulating energy homeostasis. Activation of $M C 4 R$ can decrease food intake and increase energy expenditure, thereby leading to reduce fat stores [17]. The $M C 4 R$ signaling was identified to regulate the sympathetic nerve activity and glucose tolerance [18]. In addition, the SNPs in MC4R play an important role in the susceptibility to obesity. Rare functional mutations in $M C 4 R$ are known to develop childhood-onset obesity [19-22]. Furthermore, mutations leading to complete loss of function are correlated with a more severe phenotype [23], and targeted disruption of the melanocortin-4 receptor also results in obesity in mice [24]. One study has revealed that common genetic variations near or in the MC4R contributes to obesity in American Indians [25]. However, most of GWAS loci are common variants, which located in intergenic and non-coding regions and do not alter change coding sequence [26, 27]. Earlier studies showed that GWAS-identified genetic risk for complex traits often affect phenotype by changing the mount of protein production, rather than altering the type of protein production [28]. We hypothesis the common SNP rs17782313 may influence transcriptional level of $M C 4 R$, even though the association signal located $188 \mathrm{~kb}$ downstream of the $M C 4 R$ coding sequence.

$M C 4 R$ expression are available from public TCGA database, and we obtained evidence that rs17782313 was notably associated with the expression level of $M C 4 R$.Our result showed that the expression of $M C 4 R$ in individuals carrying rs17782313 TT genotype are significantly lower than that in CT/CC genotype individuals $\left(P=1.9 \times 10^{-3}\right)$.

Given lack of direct functional evidence relating rs17782313 to $M C 4 R$ expression, we assume it alter 
the methylation level contributing to gene expression. Epigenetic, particular DNA methylation, is a crucial mechanisms of transcriptional regulation that occur without an alteration of the involving DNA sequence
$[29,30]$. Previous study indicated that gene silencing in cancer are associated with promoter Hypermethylation [31], and strongly correlations were observed between many obesity-associated SNPs and DNA methylation
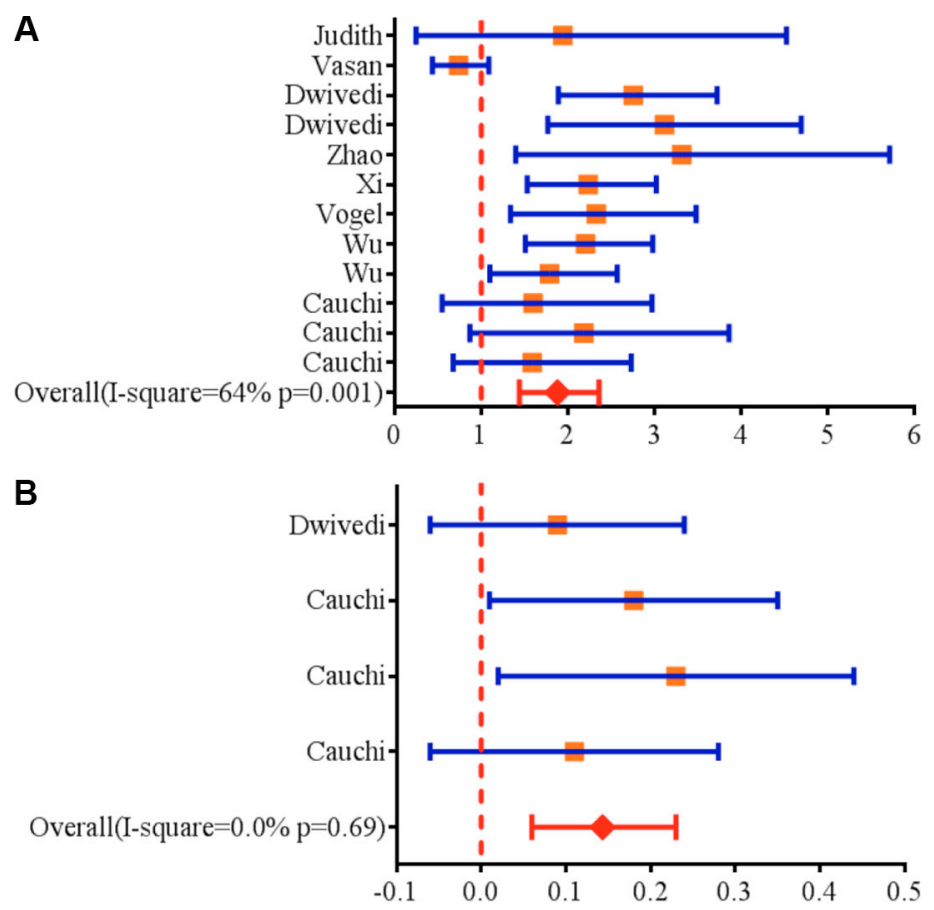

Figure 1: (A) Forest plots for the association between $M C 4 R$ rs17782313 polymorphism and genetic susceptibility to childhood obesity (CC vs. TT). Squares boxes indicate the odds ratios and the size of the box is proportional to the weight of the study. Dashed vertical lines represent the null value $(\mathrm{OR}=1.0)$. Horizontal lines represent the $95 \%$ confidence intervals. Diamonds indicate the overall summary estimate derived from a random-effects (RE) model. (B) Forest plots for the association between $M C 4 R$ rs 17782313 polymorphism and genetic susceptibility to BMI z-score (CC vs. TT). Squares boxes indicate WMD (weighted mean difference) and the size of the box is proportional to the weight of the study. Dashed vertical lines represent the null value (WMD $=0$ ). Horizontal lines represent the $95 \%$ confidence intervals. Diamonds indicate the overall summary estimate derived from a fixed-effects (FE) model.
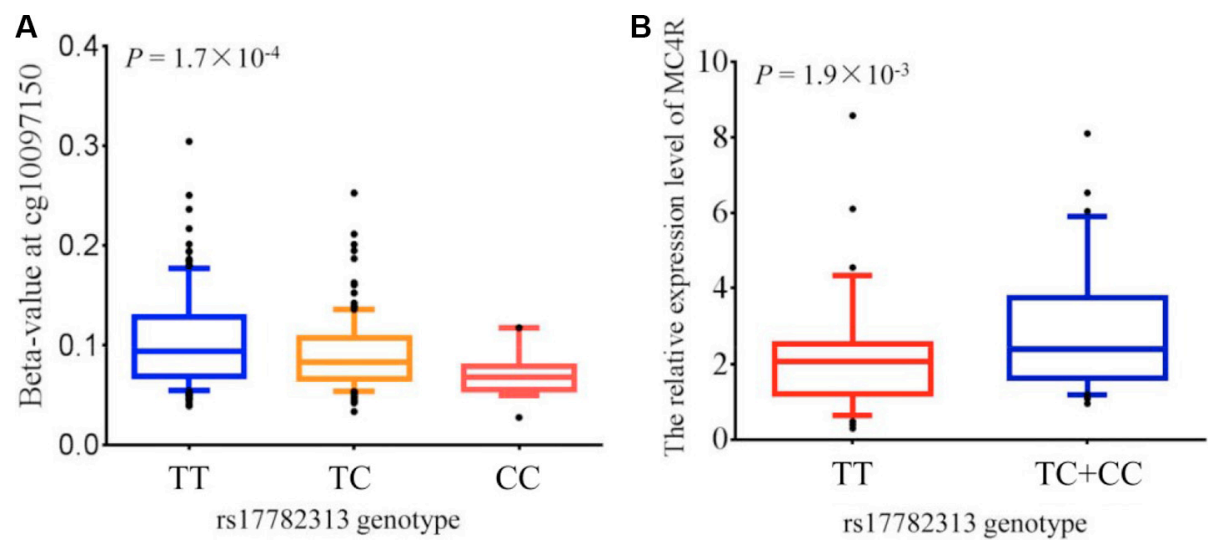

Figure 2: meQTL (methylation quantitative trait loci) and eQTLs (expression quantitative trait loci) analysis for MC4R. (A) The association between genotype at rs17782313 and methylation levels at the significant CpG probe cg10097150 which is located in the promoter region of $M C 4 R$. The level of methylation at the $\mathrm{CpG}$ probe is shown as the $\beta$-value. The box plots show the distribution of the methylation levels in each genotype category with error bars representing the $25 \%$ and $75 \%$ quantiles. (B) $M C 4 R$ expression in normal intestinal tissues stratified by genotype at rs 17782313 . The box plots show the distribution of the $M C 4 R$ expression levels in each genotype category with error bars representing the $25 \%$ and $75 \%$ quantiles. 
alternation at proximal promoters and enhancers [32]. It was reported that genotype-specific methylation in the promoter of CHRNB4 affected by SNPs that were remarkably corrected with lung cancer risk [33]. Another study found that rs $11636753 \mathrm{G}$ allele was significantly associated with decreased CHRNA5 DNA methylation, lower CHRNA5 expression and increased the risk of nicotine dependence [34].

In this study, we apply a method of integrated experiments to explore the effect of common genetic variant rs 17782313 on DNA methylation from available GEO database. Interestingly, linear regression revealed the prominent association between rs17782313 and methylation level of $\operatorname{cg} 10097150$ which is located in the promoter of $M C 4 R\left(P=1.7 \times 10^{-4}\right)$. Or rather, individuals carrying rs17782313 TT have a remarkably hypermethylation level in $M C 4 R$ promoter than those with $\mathrm{CT} / \mathrm{CC}$ genotype. Altogether, our results provide evidence that rs 17782313 may affect the methylation level of $M C 4 R$, which contribute to regulate $M C 4 R$ expression, thus involved in the development of childhood obesity.

There are some limitations in our study. First, our analysis is based on public database and studies with greater sample size are needed to validate our findings.

Additionally, the interaction between environmental exposures and genetic and epigenetic mechanisms should be considered in the following research.

In summary, our meta-analysis of data from current and published studies confirms the significant association between $M C 4 R$ polymorphism and the risk of childhood obesity and BMI z-score. Furthermore, eQTL and meQTL results strongly indicate that obesity-associated rs17782313 $\mathrm{T}$ allele was significantly associated with promoter hypermethylation and decreased expression of $M C 4 R$, thus involved in the childhood obesity. This study highlights that genetic and epigenetic mechanism in $M C 4 R$ promoter may interact and together affect functional relevance for $M C 4 R$ expression and development of childhood obesity.

\section{MATERIALS AND METHODS}

\section{Literature and search strategy}

We search relevant articles published in English from following databases, including PubMed and Embase. The search strategy to identify all possible studies involved the use of the following keywords: $(M C 4 R)$ and (polymorphism OR variant OR genotype) and (obesity). References of the retrieved articles were hand-searched.

\section{Inclusion criteria and data extraction}

The enrolled studies met the following inclusion criteria: (1) Case-control, cross-sectional, cohort studies which evaluated the association of MC4R rs17782313 with obesity. Cases diagnosed by the WHO standards of childhood obesity patients. Height-standard weight method was applied among age less than 10-year-old and BMI (Body mass index) was used for $10 \sim 18$ years old children to assess obesity levels. The control group was composed of normal healthy children; (2) Providing an odds ratio (OR) with $95 \%$ confidence interval under various inheritable models or sufficient raw data to calculate these estimates; (3) Reliable and high-quality data by using appropriate statistical analysis method; (4) If data were repetitively reported, the study with biggest sample size was included. Two authors independently assessed the articles for compliance with the inclusion criteria, and inconsistent were resolved by agreeing with the third reviewer.

\section{The cancer genome atlas (TCGA) and gene expression omnibus (GEO) databases}

Gene expression profiles were downloaded from TCGA project by RNA-Seq (level 3). In total, 434 colon adenocarcinoma tissues and 41 normal colon samples were included. To control for potential batch effects of mRNA expressions, a series of normalizations and corrections were applied. Briefly, level 3 mRNA expression of each gene was $\log 2$ transformed if it was not normally distributed, and genes with zero values were removed. We also accessed TCGA individual level 2 SNP data from tissues and blood, which were genotyped with an Affymetrix Human Genome Wide SNP 6.0 array. The genotype of rs17782313 and corresponding methylation levels were available from GEO database (GSE73103). A total of 355 healthy individuals were genotyped and obtained DNA methylation levels in their blood using the Illuminal 450K BeadChip.

\section{Statistical analysis}

Meta-analysis was conducted by STATA version 11 (StataCorp LP, College Station, TX, USA). Heterogeneity was evaluated by Cochran'Q and $I^{2}$ statistics. $P_{\text {het }} \leq 0.10$ and $I^{2}$ values $>75 \%$ were considered to be significant heterogeneity and we used randomeffect model to calculate combined effect, otherwise we applied fixed-effect model. $P<0.05$ was used as threshold to exclude genotype data which did not meet Hardy-Weinberg equivalent. Data were pooled across studies, and OR (odds ratio) and 95\%CI (confidence interval) for categorical variables and WMD (weighted mean difference) for continuous outcomes, weighted according to study sample size, were calculated. We applied linear regression to evaluate the relationship between rs17782313 risk allele and gene epigenetic and expression alterations from public GEO and TCGA database by using $\mathrm{R}$ software. All statistical analyses were two-sided, and a $P$ value $<0.05$ was considered statistically significant. 


\section{CONFLICTS OF INTEREST}

The authors declare no competing financial interests.

\section{Authors' contributions}

L.Z. and W.L. designed the study. L.Z. and B.J wrote the main manuscript. L.Z. and Y.T. analyzed the data and prepared Tables 1-2 and Figures 1-2. All authors have read and approved the manuscript.

\section{REFERENCES}

1. Mokdad AH, Ford ES, Bowman BA, Dietz WH, Vinicor F, Bales VS, Marks JS. Prevalence of obesity, diabetes, and obesity-related health risk factors, 2001. JAMA. 2003; 289:76-79.

2. Renehan AG, Tyson M, Egger M, Heller RF, Zwahlen M. Body-mass index and incidence of cancer: a systematic review and meta-analysis of prospective observational studies. Lancet. 2008; 371:569-578.

3. Troiano RP, Flegal KM. Overweight children and adolescents: description, epidemiology, and demographics. Pediatrics. 1998; 101:497-504.

4. Haslam DW, James WP. Obesity. Lancet. 2005; 366: 1197-1209.

5. Newnham JP, Pennell CE, Lye SJ, Rampono J, Challis JR. Early life origins of obesity. Obstet Gynecol Clin North Am. 2009; 36:227-244, xii.

6. A twin study of obesity. Jama. 1986; 256:2958-2959.

7. Maes HH, Neale MC, Eaves LJ. Genetic and environmental factors in relative body weight and human adiposity. Behav Genet. 1997; 27:325-351.

8. Gong J, Schumacher F, Lim U, Hindorff LA, Haessler J, Buyske S, Carlson CS, Rosse S, Buzkova P, Fornage M, Gross M, Pankratz N, Pankow JS, et al. Fine Mapping and Identification of BMI Loci in African Americans. Am J Hum Genet. 2013; 93:661-671.

9. Wen W, Cho YS, Zheng W, Dorajoo R, Kato N, Qi L, Chen CH, Delahanty RJ, Okada Y, Tabara Y, Gu D, Zhu D, Haiman CA, et al. Meta-analysis identifies common variants associated with body mass index in east Asians. Nat Genet. 2012; 44:307-311.

10. Rushton MD, Reynard LN, Young DA, Shepherd C, Aubourg G, Gee F, Darlay R, Deehan D, Cordell HJ, Loughlin J. Methylation quantitative trait locus analysis of osteoarthritis links epigenetics with genetic risk. Hum Mol Genet. 2015; 24:7432-7444.

11. Hindorff LA, Sethupathy P, Junkins HA, Ramos EM, Mehta JP, Collins FS, Manolio TA. Potential etiologic and functional implications of genome-wide association loci for human diseases and traits. Proc Natl Acad Sci USA. 2009; 106:9362-9367.
12. Rockman MV, Kruglyak L. Genetics of global gene expression. Nat Rev Genet. 2006; 7:862-872.

13. Grundberg E, Meduri E, Sandling JK, Hedman AK, Keildson S, Buil A, Busche S, Yuan W, Nisbet J, Sekowska M, Wilk A, Barrett A, Small KS, et al. Global analysis of DNA methylation variation in adipose tissue from twins reveals links to disease-associated variants in distal regulatory elements. Am J Hum Genet. 2013; 93:876-890.

14. Youngson NA, Morris MJ. What obesity research tells us about epigenetic mechanisms. Philos Trans R Soc Lond B Biol Sci. 2013; 368:20110337.

15. Loos RJ, Lindgren CM, Li S, Wheeler E, Zhao JH, Prokopenko I, Inouye M, Freathy RM, Attwood AP, Beckmann JS, Berndt SI, Jacobs KB, Chanock SJ, et al. Common variants near MC4R are associated with fat mass, weight and risk of obesity. Nat Genet. 2008; 40:768-775.

16. Krashes MJ, Lowell BB, Garfield AS. Melanocortin-4 receptor-regulated energy homeostasis. Nat Neurosci. 2016; 19:206-219.

17. Balthasar N, Dalgaard LT, Lee CE, Yu J, Funahashi H, Williams T, Ferreira M, Tang V, McGovern RA, Kenny CD, Christiansen LM, Edelstein E, Choi B, et al. Divergence of melanocortin pathways in the control of food intake and energy expenditure. Cell. 2005; 123:493-505.

18. Morgan DA, McDaniel LN, Yin T, Khan M, Jiang J, Acevedo MR, Walsh SA, Ponto LL, Norris AW, Lutter M, Rahmouni K, Cui H. Regulation of glucose tolerance and sympathetic activity by MC4R signaling in the lateral hypothalamus. Diabetes. 2015; 64:1976-1987.

19. Yeo GS, Farooqi IS, Aminian S, Halsall DJ, Stanhope RG, O'Rahilly S. A frameshift mutation in MC4R associated with dominantly inherited human obesity. Nat Genet. 1998; 20:111-112.

20. Hebebrand J, Fichter M, Gerber G, Gorg T, Hermann H, Geller F, Schafer H, Remschmidt H, Hinney A. Genetic predisposition to obesity in bulimia nervosa: a mutation screen of the melanocortin-4 receptor gene. Mol Psychiatry. 2002; 7:647-651.

21. Doulla M, McIntyre AD, Hegele RA, Gallego PH. A novel MC4R mutation associated with childhood-onset obesity: A case report. Paediatr Child Health. 2014; 19:515-518.

22. Vaisse C, Clement K, Guy-Grand B, Froguel P. A frameshift mutation in human MC4R is associated with a dominant form of obesity. Nat Genet. 1998; 20:113-114.

23. Farooqi IS, Keogh JM, Yeo GS, Lank EJ, Cheetham T, O'Rahilly S. Clinical spectrum of obesity and mutations in the melanocortin 4 receptor gene. N Engl J Med. 2003; 348:1085-1095.

24. Huszar D, Lynch CA, Fairchild-Huntress V, Dunmore JH, Fang Q, Berkemeier LR, Gu W, Kesterson RA, Boston BA, Cone RD, Smith FJ, Campfield LA, Burn P, et al. Targeted disruption of the melanocortin-4 receptor results in obesity in mice. Cell. 1997; 88:131-141. 
25. Muller YL, Thearle MS, Piaggi P, Hanson RL, Hoffman D, Gene B, Mahkee D, Huang K, Kobes S, Votruba S, Knowler WC, Bogardus C, Baier LJ. Common genetic variation in and near the melanocortin 4 receptor gene (MC4R) is associated with body mass index in American Indian adults and children. Hum Genet. 2014; 133:1431-1441.

26. Chung CC, Magalhaes WC, Gonzalez-Bosquet J, Chanock SJ. Genome-wide association studies in cancercurrent and future directions. Carcinogenesis. 2010; 31:111-120.

27. Rafnar T, Sulem P, Stacey SN, Geller F, Gudmundsson J, Sigurdsson A, Jakobsdottir M, Helgadottir H, Thorlacius S, Aben KK, Blondal T, Thorgeirsson TE, Thorleifsson G, et al. Sequence variants at the TERT-CLPTM1L locus associate with many cancer types. Nat Genet. 2009; 41:221-227.

28. Nicolae DL, Gamazon E, Zhang W, Duan S, Dolan ME, Cox NJ. Trait-associated SNPs are more likely to be eQTLs: annotation to enhance discovery from GWAS. PLoS Genet. 2010; 6:e1000888.

29. Kulis M, Heath S, Bibikova M, Queiros AC, Navarro A, Clot G, Martinez-Trillos A, Castellano G, Brun-Heath I, Pinyol M, Barberan-Soler S, Papasaikas P, Jares P, et al. Epigenomic analysis detects widespread gene-body DNA hypomethylation in chronic lymphocytic leukemia. Nat Genet. 2012; 44:1236-1242.
30. Liu Y, Aryee MJ, Padyukov L, Fallin MD, Hesselberg E, Runarsson A, Reinius L, Acevedo N, Taub M, Ronninger M, Shchetynsky K, Scheynius A, Kere J, et al. Epigenomewide association data implicate DNA methylation as an intermediary of genetic risk in rheumatoid arthritis. Nat Biotechnol. 2013; 31:142-147.

31. Herman JG, Baylin SB. Gene silencing in cancer in association with promoter hypermethylation. N Engl J Med. 2003; 349:2042-2054.

32. Voisin S, Almen MS, Zheleznyakova GY, Lundberg L, Zarei S, Castillo S, Eriksson FE, Nilsson EK, Bluher M, Bottcher Y, Kovacs P, Klovins J, Rask-Andersen M, et al. Many obesity-associated SNPs strongly associate with DNA methylation changes at proximal promoters and enhancers. Genome Med. 2015; 7:103.

33. Scherf DB, Sarkisyan N, Jacobsson H, Claus R, Bermejo JL, Peil B, Gu L, Muley T, Meister M, Dienemann H, Plass C, Risch A. Epigenetic screen identifies genotype-specific promoter DNA methylation and oncogenic potential of CHRNB4. Oncogene. 2013; 32:3329-3338.

34. Hancock DB, Wang JC, Gaddis NC, Levy JL, Saccone NL, Stitzel JA, Goate A, Bierut LJ, Johnson EO. A multiancestry study identifies novel genetic associations with CHRNA5 methylation in human brain and risk of nicotine dependence. Hum Mol Genet. 2015; 24:5940-5954. 